${ }^{1} \mathrm{~S}$ M Mattioli, ${ }^{2}$ Sauni, ${ }^{3}$ Spreeuwers, ${ }^{4}$ De Schryver, ${ }^{5}$ Valenty, ${ }^{5}$ Rivière, ${ }^{1}$ Curti. ${ }^{1}$ University of Bologna, Bologna, Italy; ${ }^{2}$ Finnish Institute of Occupational Health, Tampere, Finland; ${ }^{3}$ SanEcon Ltd, Amersfoort, The Netherlands; ${ }^{4}$ Epidemiology and Social Medicine, University of Antwerp, Antwerpen, Belgium; ${ }^{5}$ Département Santé au travail, Institut de Veille Sanitaire, Saint Maurice, France

\subsection{6/oemed-2013-101717.203}

Objectives Underreporting of occupational diseases (OD) is an important issue worldwide. To address this problem, we projected a Cochrane review to evaluate the effect of interventions aimed at reducing the underreporting of OD by physicians.

Methods We will include randomised controlled trials (RCT), cluster-RCT, controlled before-and-after (CBA) and interrupted time-series (ITS) studies. We will include any type of intervention acting directly or indirectly to influence the behaviour of physicians. As primary outcome, we will define the reporting of OD either measured as the number of physicians reporting or as the number of OD reported per physician. Pairs of authors will independently screen the titles and abstracts of the search strategy results. Potentially relevant articles will be obtained in full text and independently assessed for inclusion.

Results A preliminary search to locate RCT was conducted in Medline (through Pubmed) up to November 2012. The search strategy identified 137 potentially pertinent articles. Of these, three articles met the inclusion criteria. Two RCT were conducted in high income countries (the Netherlands and United States), while the other one was conducted in Nigeria. Two studies evaluated the effect of informative interventions, the third one evaluated the effect of a training programme. Results indicate insufficient evidence for the effect of informative interventions for reducing the underreporting of OD (1 RCT on occupational physicians, not effective - 1 RCT on physicians, effective). On the other hand, training had a positive effect on health personnel knowledge, reporting requirement and the timeliness and completeness of the disease surveillance and notification system.

Conclusions These preliminary results, not including data from future searches regarding CBA and ITS, highlight the widespread problem of underreporting of OD. More high quality RCT are needed to evaluate the effect of interventions which could be applied to increase the reporting of OD in different contexts and countries.

\section{SEARCHING FOR SIGNALS OF POTENTIALLY NEW DISEASE-EXPOSURE ASSOCIATIONS: INTEREST OF SCREENING WORK-RELATED DISEASES SURVEILLANCE DATABASES WITH DATA MINING APPROACHES?}

${ }^{1} \mathrm{~V}$ B Bonneterre, ${ }^{2}$ Bicout, ${ }^{3}$ Agius, ${ }^{2}$ De Gaudemaris. ${ }^{1} \mathrm{CHU}$ Grenoble (Teaching Hospital), Grenoble Cedex 9, France; ${ }^{2}$ UJF-Grenoble 1/CNRS/TIMC-IMAG UMR 5525 (EPSP team), Grenoble, France; ${ }^{3}$ Centre for Occupational and Environmental Health, The University of Manchester, Manchester, United Kingdom

\subsection{6/oemed-2013-101717.204}

Objective We defined potential new work-related diseases as either new couples "disease $\mathrm{x}$ agent", or new triads "disease $\mathrm{x}$ agent $\mathrm{x}$ occupational setting" ("old friends in new places") with, at least, a reasonably strong causality presumption. Before such cases have been reported a high number of times and raise clinicians' concern, they will have been encountered only a few times, sometimes by different physicians. Some cases might have been captured by surveillance networks. Thus, it is for interest for these networks to develop tools trying to highlight pro-actively these kinds of early potential signals within their growing databases (rather than waiting to be asked, after clinician concern was expressed, how many cases they have been recorded the previous years). The objective of this communication, is to show and illustrate to what extent, a Data Mining approach could help identifying such cases of interest for vigilance purposes.

Methods Databases from the French National Surveillance Scheme on occupational diseases Surveillance and Prevention (rnv3p), and from the UK team of occupational diseases surveillance (THOR) have been explored (both schemes being part of a wider Modernet consortium, whose networking is currently funded by EU-COST program).

Analyses of the existing couples and triads and identification of those generating a signal with disproportionality measures used in pharmacovigilance (ex PRR: Proportional Reporting Ratio).

Results New couples and triads already derived from these analyses conducted on rnv3p and THOR schemes will be presented. Conclusion These methods stand at the frontier of conventional epidemiological surveillance of work-related diseases, and might be beneficial for vigilance in highlighting similar cases, in order to investigate them as early as possible. The higher the "background noise" in the database, the more efficient they are to highlight disproportionalities.

\section{Session: Mini symposium V: Shift work and cancer}

\section{BIOLOGICAL MECHANISMS THAT UNDERLIE SHIFTWORK AS A RISK FACTOR FOR BREAST CANCER}

${ }^{1} \mathrm{~L}$ Fritschi, ${ }^{2} \mathrm{~T}$ Erren, ${ }^{3} \mathrm{D}$ Glass, ${ }^{1} \mathrm{C}$ Saunders, ${ }^{1} \mathrm{~J}$ Girschik, ${ }^{1} \mathrm{~T}$ Boyle, ${ }^{1} \mathrm{~S}$ El-Zaemey, ${ }^{1} \mathrm{~A} K$ K Thomson, ${ }^{1} \mathrm{P}$ Rogers, ${ }^{1} \mathrm{~S}$ Peters, ${ }^{4} \mathrm{~T}$ Slevin, ${ }^{1} \mathrm{~A}$ D'Orsogna, ${ }^{5} \mathrm{~F}$ De Vocht, ${ }^{1} \mathrm{~J}$ Heyworth. ${ }^{1}$ University of Western Australia, Perth, Australia; ${ }^{2}$ Cologne University, Cologne, Germany; ${ }^{3}$ Monash University, Melbourne, Australia; ${ }^{4}$ Cancer Council of WA, Perth, Australia; ${ }^{5}$ The University of Manchester, Manchester, United Kingdom

\subsection{6/oemed-2013-101717.205}

Objectives We investigated hypotheses for the association between shiftwork and breast cancer based on our a priori theoretical framework of five biological mechanisms which might be operational in shiftwork: light at night; phase shift (when central cycles have adjusted to night work, but peripheral cycles have not); sleep disruption; lifestyle factors (diet, physical activity and alcohol intake) and low vitamin D.

Methods We conducted a population-based case-control study with 1205 breast cancer cases, identified from 2009 to 2011 identified through the Western Australian Cancer Registry, and 1789 age-matched controls from the Western Australian electoral roll. An occupational history was collected by self-completed questionnaire for every job a woman had held for at least six months (job title, main tasks, year started, duration, hours per week and weeks per year worked, and whether the job involved night work, shift work, or work at unusual hours). Using the web application OccIDEAS, we obtained further details about the shiftwork by telephone interview. Automatic assessments with manual reviews were used to assess occupational exposure to the hypothesized factors.

Results We found a $22 \%$ increase in breast cancer risk (OR $1 \cdot 22,95 \%$ CI 1.01-1.47) for phase shift with a statistically significant dose response relationship $(\mathrm{p}=0.04)$. For the other hypothesized mechanisms, risks were marginally elevated and 